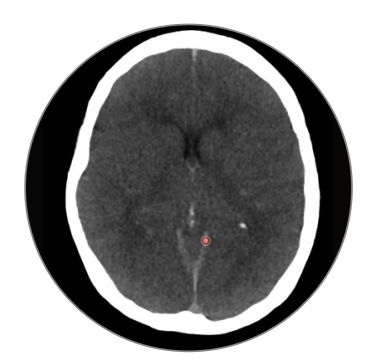

\section{Pseudohemorragia subaracnoidea. Presentación de un caso}

\author{
Pseudo-subarachnoid Hemorrhage. A Case Report
}

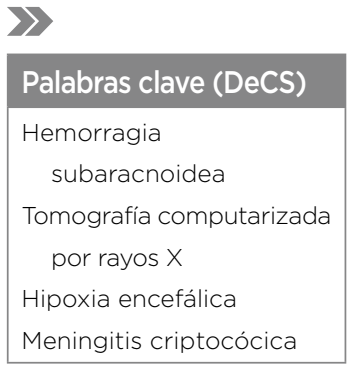

\section{Key words (MeSH)}

Subarachnoid

hemorrhage

Tomography, X-ray

computed

Hypoxia, brain

Cryptococcal meningitis

'Neurólogo, Pontificia Universidad Javeriana. Neurólogo y docente, Hospital Universidad del Norte. Barranquilla, Colombia.

${ }^{2}$ Residente de Radiología e imágenes diagnósticas, tercer año, Universidad del Norte, Hospital Universidad del Norte. Barranquilla, Colombia.

${ }^{3}$ Médica general, Universidad del Norte, Hospital Universidad del Norte. Barranquilla, Colombia

${ }^{4}$ Residente de Medicina interna, segundo año, Universidad del Norte, Hospital Universidad del Norte. Barranquilla, Colombia.

${ }^{5}$ Residente de Medicina interna, primer año, Universidad del Norte, Hospital Universidad del Norte. Barranquilla, Colombia.

${ }^{6}$ Residente de Medicina interna, segundo año. Universidad del Norte, Hospital Universidad del Norte. Barranquilla, Colombia.

Departamento de Radiología e Imágenes Diagnósticas. Hospital Universidad del Norte.

\section{Resumen}

La pseudohemorragia subaracnoidea es un fenómeno infrecuente que se caracteriza por hallazgos sugestivos de hemorragia subaracnoidea en la tomografía computarizada simple de cráneo, sin evidencia de la misma en estudios adicionales. Se ha asociado a múltiples causas, de las cuales la principal es la encefalopatía hipóxico-isquémica posparo cardiaco y reanimación cardiopulmonar. El contexto clínico y los niveles de atenuación medidos en Unidades Hounsfield (UH) se deben tener en cuenta al hacer el diagnóstico diferencial entre ambas entidades. Se presenta el caso de una paciente con pseudohemorragia subaracnoidea de etiología multifactorial.

\section{Summary}

Pseudo-subarachnoid hemorrhage (PSAH) is an infrequent entity characterized by findings in noncontrast head computed tomography that mimic subarachnoid hemorrhage, but without evidence of blood products in further studies. It has been associated with multiple etiologies, with hypoxic ischemic encephalopathy following cardiac arrest and cardiopulmonary resuscitation as the leading cause in literature. Clinical context and attenuation levels measured in Hounsfield Units should be taken into consideration when establishing the differential diagnosis between these entities. The case of a patient with PSAH of multifactorial etiology is presented.

\section{Introducción}

La pseudohemorragia subaracnoidea es una entidad caracterizada por hallazgos tomográficos que imitan la hemorragia subaracnoidea (HSA), sin evidencia de sangrado en estudios adicionales, como los realizados en el líquido cefalorraquídeo (LCR) o el material de autopsia. En la tomografía axial computarizada (TAC) de cráneo se observa aumento simétrico de la densidad en las cisternas de la base, las cisternas silvianas y el espacio subaracnoideo, por lo que suele reseñarse como HSA. Se ha asociado a múltiples causas, entre ellas encefalopatía hipóxico-isquémica luego de un paro cardiaco y reanimación cardiopulmonar (RCP), edema cerebral difuso, leptomeningitis piógena e hipotensión intracraneana, entre otros (1).

\section{Presentación del caso}

Se trata de una paciente de 59 años, con antecedente de cirrosis hepática por hepatitis crónica y sospecha de hepatocarcinoma. Ingresó por cuadro clínico de 20 días de evolución de cefalea asociada a hipoacusia derecha, disminución de la agudeza visual, disartria y dificultad para la deglución y la marcha. Al ingreso se encontró alerta, orientada, con signos vitales dentro de límites normales. Se evidenció bradipsiquia, bradilalia, ataxia troncular y neuropatía craneal múltiple por compromiso de pares craneanos III, VI, VIII, IX, X y XII. Los exámenes de laboratorio demostraron hiperbilirrubinemia, elevación de transaminasas, trombocitopenia e hiponatremia. En ecografía abdominal se observaron cambios por hepatopatía crónica y masas hepáticas altamente sugestivas de hepatocarcinoma (LI-RADS-US 3B), tomografía axial computada (TAC) de cráneo con medio de contraste cuyo resultado estuvo dentro de límites normales (figura 1) y punción lumbar. Los resultados del estudio del LCR fueron: leucocitos 0 , eritrocitos $180 / \mathrm{mm}^{3}$ (90\% frescos y $10 \%$ crenados, posiblemente por punción traumática), glucosa $23 \mathrm{mg} / \mathrm{dL}$, proteínas $126 \mathrm{mg} / \mathrm{dL}$, Gram, Z-N y tinta china negativos y FilmArray positivo para criptococo. Se diagnosticó criptococosis meníngea y se inició tratamiento para la misma. Al quinto día de estancia hospitalaria, presentó deterioro del estado de consciencia y paro cardiorrespiratorio. Se realizó RCP con retorno de la circulación espontánea a los 8 minutos. Se trasladó a la unidad de cuidado intensivo con soporte vasopresor. $\mathrm{Su}$ examen neurológico revelaba escala de Glasgow 3/15 y ausencia de reflejos de tallo. Se realizó TAC de cráneo simple en el que se evidenció edema cerebral grave caracterizado por pérdida de la diferenciación de las sustancias gris y blanca y pérdida de los surcos, asociado a un aumento simétrico de la densidad en las cisternas 
de la base, con valores densitométricos entre 33-46 UH, compatible con pseudohemorragia subaracnoidea (figuras 2 y 3); 48 horas después tuvo mayor deterioro hemodinámico y paro cardiorrespiratorio, posterior a lo cual se declaró su defunción.

\section{Discusión}

El signo de la pseudohemorragia subaracnoidea fue descrito por primera vez por Spiegel et al. en 1986 (2). Informaron 10 casos de pacientes con hallazgos tomográficos sugestivos de HSA y edema cerebral, sin evidencia de esta en las autopsias. En 1998, Avrahami et al. realizaron una revisión de 100 pacientes con tomografias craneales compatibles con HSA, sin evidenciar sangre ni xantocromía en LCR (3).

La pseudohemorragia subaracnoidea se caracteriza por baja densidad del parénquima cerebral en la TAC simple, que puede asociarse a alta densidad de la vasculatura, imitando sangre en las cisternas basales y el espacio subaracnoideo. Cursa con alta densidad en surcos y cisternas, pero sin evidencia clínica o patológica que la apoye, por lo que suele interpretarse como un falso positivo de HSA. Además, se observa pérdida de la diferenciación entre la sustancia gris y blanca con borramiento de las cisternas basales, lo que indica edema cerebral difuso (4). A diferencia de la HSA aneurismática, que se asocia típicamente a cefalea "tipo trueno", en la pseudohemorragia subaracnoidea las manifestaciones clínicas son variables, ya que puede asociarse a múltiples etiologías (5).

Generalmente, el valor de la atenuación medido en Unidades Hounsfield (UH) es útil para distinguir la pseudohemorragia subaracnoidea de la HSA. En la pseudohemorragia subaracnoidea varía entre 30-45 UH, mientras que en la HSA aneurismática, al tratarse de productos sanguíneos, suele variar entre 60-70 UH $(6,7)$.

La fisiopatología de la pseudohemorragia subaracnoidea depende de su etiología. Cuando es infecciosa, la producción de toxinas puede causar alteración de la barrera hematoencefálica y permitir el paso de proteínas al espacio subaracnoideo, lo que puede aumentar el coeficiente de atenuación e imitar una HSA en casos graves. Cuando se asocia a edema cerebral difuso, suele encontrarse alteración de la barrera hematoencefálica y edema vasogénico. En la fase aguda de una lesión cerebral, la reabsorción del LCR resuelve parcialmente el edema y, aunque el contenido de proteínas en LCR puede estar elevado, no modifica de manera significativa su valor de atenuación en la TAC (4). Sin embargo, cuando el edema es grave, aumenta la presión intracraneal (PIC) y pueden comprimirse los senos venosos durales, lo que afecta el drenaje venoso y de LCR. Como consecuencia, se produce congestión y dilatación de las venas superficiales, que resaltan contra un parénquima de baja densidad, imitando así la HSA. Además, el aumento de la PIC y la inflamación reducen el espacio subaracnoideo, desplazando el LCR y aumentando la proporción de meninges y vasos sanguíneos, lo que contribuye al aumento de su atenuación (4).

La paciente manifestaba cefalea con signos de alarma por neuropatía craneal múltiple asociada a hepatopatía crónica y probable hepatocarcinoma. Se diagnosticó criptococosis meníngea mediante la prueba FilmArray del LCR, la cual tiene una especificidad del 99,7\% para este diagnóstico (8). En la TAC de control post-RCP se evidenciaron los hallazgos previamente descritos, compatibles con pseudohemorragia subaracnoidea en un contexto clínico altamente sugestivo de la misma (estado post-RCP, TAC inicial sin hallazgos patológicos y LCR no sugestivo de HSA). Entre las posibles causas de pseudohemorragia subaracnoidea en esta paciente se encuentra la neuroinfección, que puede alterar la permeabilidad de la

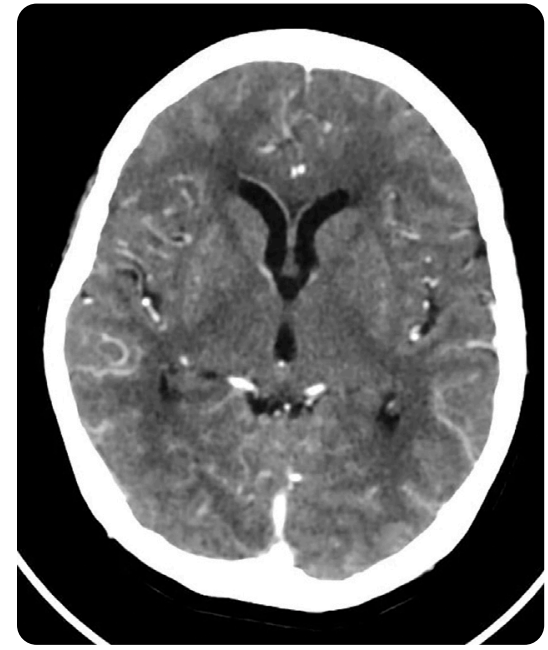

Figura 1. TAC de cráneo con medio de contraste, corte axial, realizado al ingreso. No se observan hallazgos patológicos.

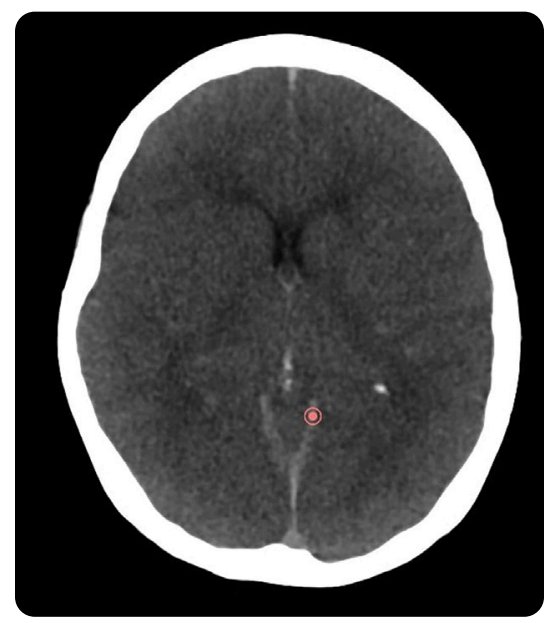

Figura 2. TAC de cráneo simple, corte axial. Se observa pérdida de la diferenciación entre la sustancia gris y blanca, con disminución de la profundidad de los surcos. El puntero señala un aumento de la densidad en el espacio subaracnoideo en la tienda del cerebelo.

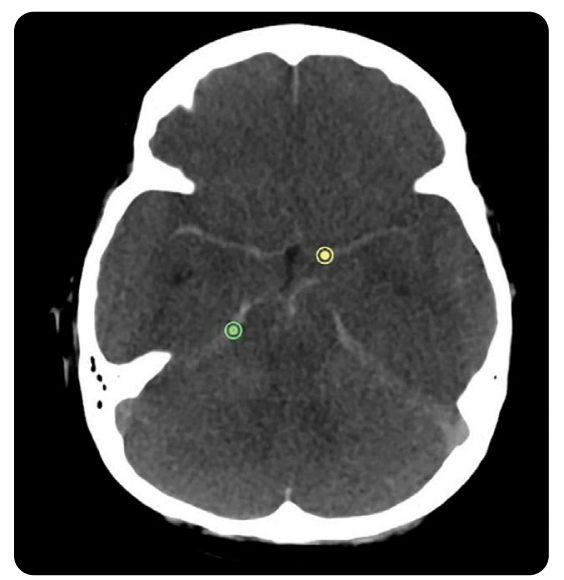

Figura 3. TAC de cráneo simple, corte axial. Se observa pérdida de la diferenciación entre la sustancia gris y blanca, con disminución de la profundidad de los surcos. Los punteros señalan un aumento de la densidad en el espacio subaracnoideo en las cisternas de la base. 
barrera hematoencefálica y producir edema vasogénico. Sin embargo, debido a la cronología de las manifestaciones clínicas e imagenológicas, se considera que los hallazgos se deben principalmente a encefalopatía hipóxico-isquémica post-RCP.

\section{Conclusión}

La pseudohemorragia subaracnoidea es de peor pronóstico que la HSA y se ha asociado a desenlaces neurológicos adversos. Por lo tanto, es de vital importancia conocer su patrón radiológico e incluirla dentro del diagnóstico diferencial cuando el contexto clínico lo sugiere, ya que su pronta identificación puede facilitar la toma de decisiones con respecto al final de la vida y la realización de procedimientos invasivos.

\section{Bibliografía}

1. Ferrante E, Regna-Gladin C, Arpino I, et al. Pseudo-subarachnoid hemorrhage: a potential imaging pitfall associated with spontaneous intracranial hypotension. Clin Neurol Neurosurg. 2013;115(11):2324-8.

2. Spiegel S, Fox A, Vinuela F, et al. Increased density of tentorium and falx: a false positive CT sign of subarachnoid hemorrhage. Can Assoc Radiol J. 1986;37(4):243-7.

3. Avrahami E, Katz R, Rabin A, et al. CT diagnosis of non-traumatic subarachnoid haemorrhage in patients with brain edema. Eur J Radiol. 1998;28(3):222-5.

4. Given C, Burdette J, Elster A, et al. Pseudo-subarachnoid hemorrhage: A potential imaging pitfall associated with diffuse cerebral edema. Am J Neuroradiol. 2003;24(2):254-6.

5. Hasan T, Duarte W, Akinduro O, et al. Nonaneurysmal "pseudo-subarachnoid hemorrhage" computed tomography patterns: challenges in an acute decision-making heuristics. J Stroke Cerebrovasc Dis. 2018;27(9):2319-26.

6. Yuzawa H, Higano S, Mugikura S, et al. Pseudo-subarachnoid hemorrhage found in patients with postresuscitation encephalopathy: characteristics of CT findings and clinical importance. Am J Neuroradiol. 2008;29(8):1544-9.

7. Senthilkumaran S, Balamurugan N, Menezes R, et al. Role of Hounsfield units to distinguish pseudo-subarachnoid hemorrhage. Clin Toxicol. 2011;49:948.

8. O'Halloran J, Franklin A, Lainhart W, et al. Pitfalls associated with the use of molecular diagnostic panels in the diagnosis of cryptococcal meningitis. Open Forum Infect Dis. 2017;4(4):ofx242.

\section{Correspondencia}

Laura López Brokate

Carrera 56 \# 79-342

Barranquilla, Colombia

lbrokate@uninorte.edu.co

Recibido para evaluación: 26 de octubre de 2020

Aceptado para publicación: 19 de agosto de 2020 